\title{
Etude de l'activité nitrogénase des nodosités de soja inoculé avec une souche à croissance rapide de Rhizobium
}

\author{
Vu Ti THU, Jean-Jacques DREVON $\left({ }^{*}\right)\left({ }^{1}\right) \&$ Louis SALSAC $(*)$ \\ Ecole d'Agriculture, Truong Dai Hoc Nong Nghiep I Hanoï, Vietnam. \\ (*) I.N.R.A., Laboratoire de Recherches sur les Symbiotes des Racines, 9, place Viala, 34060 Montpellier \\ Cedex France.
}

RÉSUMÉ Les capacités fixatrices d'azote de la souche à croissance rapide Rhizobium GA et d'une souche à croissance
lente $R$. japonicum PJ17 ont été comparées sur 3 cultivars de soja de différentes origines.
En symbiose avec GA, la croissance des plantes est plus ou moins ralentie selon le cultivar.
La masse et les activités de réduction de l'acétylène, d'émission d'hydrogène et d'incorporation d'azote ${ }^{15} \mathrm{~N}$
des nodosités formées par GA et par PJ17 sont comparables.
L'apport d'un complément d'azote minéral n'augmente pas la croissance des plantes inoculées avec la souche GA.

Mots clés additionnels : Fixation de l'azote, hydrogénase.

Abréviations : ARA : activité de réduction de l'acétylène ; Hup : "hydrogen uptake "; MS : matière sèche; $\mathrm{MF}$ : matière fraîche ; meq : milliequivalent ; $\mathrm{ERa}$ : efficacité relative apparente.

Nodule nitrogenase activity of soybean inoculated with a fast-growing strain of Rhizobium.

The nitrogen-fixation capacities of the fast-growing Rhizobium GA and the slow-growing $R$. japonicum PJ17 were compared on 3 cultivars of soybean from various origins. In symbiosis with GA, plant growth was limited, the limitation intensity depending on the cultivar. The mass and the activities of acetylene reduction, hydrogen evolution and ${ }^{15} \mathrm{~N}$ incorporation of nodules formed by GA and PJ17 were not significantly different. The supply of additional mineral nitrogen did not increase growth of plants inoculated with GA.

Additional key words : Nitrogen fixation, hydrogenase.

Abbreviations : ARA : acetylene reducing activity ; Hup : hydrogen uptake ; MS : dry matter ; MF : fresh matter ; meq : milliequivalent ; ERa : apparent relative efficiency.

\section{INTRODUCTION}

L'étude de souches de Rhizobium à croissance rapide capables de noduler le soja (KEYSER et al., 1982) suscite un vif intérêt :

- pour des raisons biologiques: la symbiose Glycine max - Rhizobium japonicum est très spécifique et jusqu'à une date récente la nodulation du soja par des Rhizobium à croissance rapide était inconnue ;

- pour des raisons agronomiques : la croissance plus rapide de ces souches pourrait leur conférer une

( ${ }^{1}$ A qui les demandes de tirés à part peuvent être adressées. compétitivité supérieure à celle des Rhizobium à croissance lente pour l'infection des racines de soja ;

- pour des raisons méthodologiques : les études nécessitant des cultures répétées de bactéries donnent plus rapidement des résultats lorsque la vitesse de croissance du microorganisme est plus élevée ;

- pour des raisons économiques : la production d'inoculum est moins coûteuse et les risques de contamination sont diminués quand la vitesse de croissance du Rhizobium est accrue.

D'après des premiers travaux, ces souches à croissance rapide, auraient une faible capacité à fixer l'azote en symbiose avec les cultivars de soja sélectionnés aux Etats-Unis; au contraire, leur association avec des variétés sélectionnées en Chine présente une 
bonne capacité de fixation de l'azote (KEYSER et al., 1982).

Des souches de Rhizobium à croissance rapide ont été récemment isolées dans des sols vietnamiens. L'une d'entre elles a été introduite dans des essais comparatifs avec une souche de $R$. japonicum à croissance lente ayant une capacité élevée de fixation de l'azote. La croissance des parties aériennes et l'activité fixatrice d'azote ont été mesurées sur les symbioses de ces souches avec des variétés de soja de diverses origines géographiques.

\section{MATÉRIEL ET MÉTHODES}

\section{A. Souches de Rhizobium}

La souche à croissance rapide, dénommée GA dans la collection de notre laboratoire, a été isolée au Vietnam par l'INSA (Hanoï).

La souche PJ17 est un mutant dépourvu d'hydrogénase de la souche USDA 122 de la collection de Beltsville (LEPO et al., 1981).

Les inoculum de ces souches sont obtenus sur milieu YEM liquide à $28^{\circ} \mathrm{C}$ (VINCENT, 1970) en $48 \mathrm{~h}$, pour GA et en $8 \mathrm{j}$ pour $\mathrm{PJ} 17$.

\section{B. Variétés de soja}

Les cultivars « Giesen » et « 106-17 » ont été sélectionnés à partir de matériel d'origine américaine, le premier ayant été obtenu en Allemagne, le second en France. Le cv. «Fengshou » est d'origine asiatique.

\section{Culture des plantes}

Les graines sont stérilisées dans l'hypochlorite de calcium $\left(67 \mathrm{~g} \mathrm{.1} \mathrm{1}^{-1}\right)$ pendant $30 \mathrm{mn}$, puis lavées à l'eau distillée stérile et mises à germer stérilement sur bactoagar $(1 \mathrm{p} .100)$, à $28^{\circ} \mathrm{C}$. $48 \mathrm{~h}$ plus tard, les germes d'un lot homogène sont incubés pendant $30 \mathrm{mn}$ dans l'inoculum de Rhizobium; ils sont ensuite transférés sous serre en pots de PVC $(V=51)$. Ceux-ci sont arrosés tous les $2 \mathrm{j}$ en alternance avec de l'eau distillée ou la solution nutritive suivante : $\mathrm{KH}_{2} \mathrm{PO}_{4}: 0,1 \mathrm{mM}$; $\mathrm{CaCl}_{2}: 1,9 \mathrm{mM} ; \mathrm{MgSO}_{4}: 1,0 \mathrm{mM} ; \mathrm{KCl}: 2,9 \mathrm{mM}$; $\mathrm{H}_{3} \mathrm{BO}_{3}: 7,75 \cdot 10^{-3} \mathrm{mM} ; \mathrm{CuSO}_{4}: 0,15 \cdot 10^{-3} \mathrm{mM}$; $\mathrm{MnSO}_{4}: 0,15 \cdot 10^{-3} \mathrm{mM} ; \mathrm{ZnSO}_{4}: 0,60 \cdot 10^{-3} \mathrm{mM}$; $\mathrm{NaMoO}_{4}: 0,05 \cdot 10^{-3} \mathrm{mM} ; \mathrm{CoCl}_{2}: 0,10 \cdot 10^{-3} \mathrm{mM}$; $\mathrm{NiCl}_{2}: 0,10 \cdot 10^{-3} \mathrm{mM}$. Avant chaque arrosage, le sol est lessivé à l'eau distillée afin d'éliminer toute accumulation d'ions minéraux et d'exsudats racinaires. La photopériode est de $16 \mathrm{~h}$ de lumière naturelle complémentée par un éclairage artificiel (lampes OSRAM $1000 \mathrm{~W}: 200 \mu \mathrm{E} \mathrm{m}^{-2} \cdot \mathrm{h}^{-1}$ ) et de $8 \mathrm{~h}$ nocturnes ; la température diurne est de $28^{\circ} \mathrm{C}$ et la température nocturne de $20{ }^{\circ} \mathrm{C}$. L'humidité relative de l'atmosphère est de 70 p. 100.

\section{Mesure des activités nitrogénase et hydrogénase}

Les mesures de dégagement d'hydrogène et de réduction d'acétylène par les nodosités ainsi que l'activité hydrogénase des bactéroïdes sont réalisées comme décrit précédemment (DREVON et al., 1983). L'ERa (efficacité relative apparente) des nodosités est calculée selon la formule proposée par SCHUBERT \& EVANS (1976) :

$$
\text { ERa }=1-\frac{\text { vitesse de dégagement de } \mathrm{H}_{2}}{\text { vitesse de réduction de } \mathrm{C}_{2} \mathrm{H}_{2}} .
$$

L'incorporation de ${ }^{15} \mathrm{~N}_{2}$ est mesurée après $90 \mathrm{mn}$ d'incubation de racines nodulées excisées placées dans une atmosphère contenant initialement 20 p. $100 \mathrm{O}_{2}$, 10 p. $100 \mathrm{~A}_{\mathrm{r}}$ et 70 p. $100^{15} \mathrm{~N}_{2}$ (enrichi à 99,5 p. 100). La teneur en azote total des nodosités et des racines est déterminée par la méthode de NESLER (après diffusion en cellule de CONWAY d'un aliquot de l'échantillon minéralisé, RoBIN, 1979). L'excès en ${ }^{15} \mathrm{~N}$ est mesuré par spectrométrie optique (GUIRAUD \& FARDEAU, 1980).

Le poids des parties aériennes est mesuré après séchage pendant $48 \mathrm{~h}$ à $80^{\circ} \mathrm{C}$. La matière fraîche des nodosités est pesée aussitôt après les incubations.

\section{RÉSULTATS}

\section{A. Croissance et chlorose des plantes}

Dans les expériences où les plantes ne reçoivent aucun apport d'azote combiné, on observe un ralentissement de la croissance des plantes inoculées avec la souche GA par rapport à celle des mêmes cultivars inoculés avec PJ17 (fig. 1). Ce ralentissement est plus ou moins prononcé selon la plante hôte. Au terme de l'expérience, quand les plantes inoculées avec PJ17 sont en pleine floraison, le poids des plantes inoculées avec GA est égal à 75 p. 100 de celui des plantes inoculées avec PJ17 pour la variété «Fengshou ». Ce pourcentage est de 60 p. 100 pour «106-17», et de 42 p. 100 pour «Giesen ». Dans ce dernier cas, les plantes inoculées avec GA présentent une chlorose prononcée de leur feuillage.

L'apport après le $30^{\mathrm{e}} \mathrm{j}$ d'une dose de $1 \mathrm{meq} \mathrm{NO}_{3}^{-}$, qui n'inhibe pas la nodulation (KIMOU, 1984), ne fait pas disparaître les symptômes de chlorose observés dans l'association GA-Giesen et ne provoque aucune augmentation significative de croissance chez cette plante (tabl. 1). Au contraire, le cultivar " Fengshou » inoculé avec GA présente une croissance accrue en présence d'azote combiné. Mais, dans les 2 cas, la masse de matière sèche aérienne des plantes est inférieure à celle du même hôte inoculé avec la souche PJ17.

\section{B. Nodulation, activité nitrogénase}

Les masses de nodosités formées par GA et PJ17 sont comparables quel que soit le cultivar (fig. 1). Les nodosités formées par GA ont un poids moyen unitaire légèrement inférieur, mais leur nombre est plus élevé, d'où les masses comparables de tissu fixateur dans les différentes associations.

En début de cycle végétatif et à la floraison, l'activité nitrogénase (ARA) des nodosités formées par GA n'est pas significativement inférieure à celle des nodosités formées par la souche à croissance lente (tabl. 2). 

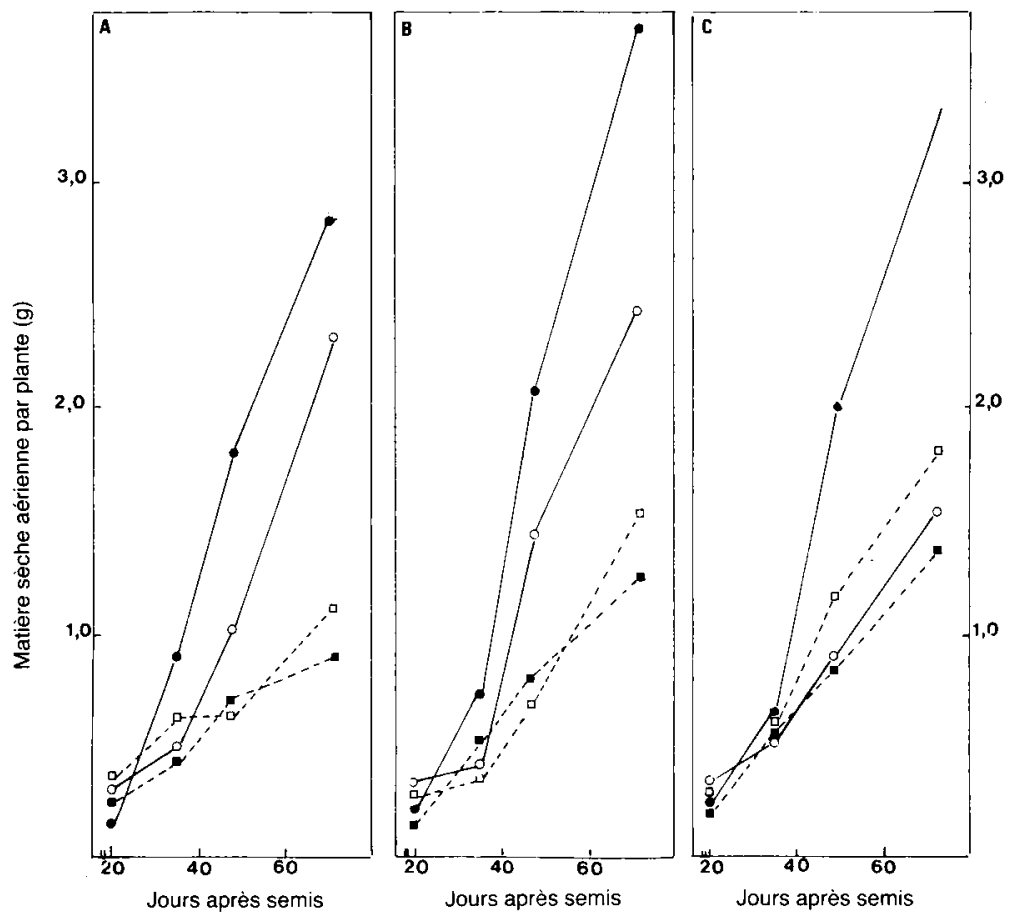

Figure 1

Croissance ( $\bullet, O)$ et nodulation $(\square, \square)$ du soja nodulé par $G A$

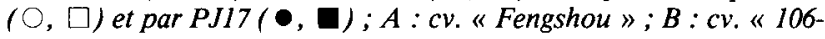
$17 » ; C: c v$. "Giesen ».

Growth ( $\bullet, O)$ and nodulation $(\boldsymbol{\square}, \square)$ of soybean nodulated by $G A(O, \square)$ and by $P J I 7(\bullet, \square) ; A: c v$. 'Fengshou'; $B: c v$. '106-17'; $C: c v$. 'Giesen'.
Chaque valeur est la moyenne de 4 répétitions par association à chaque date de prélèvement. Les $1^{\text {res }}$ fleurs apparaissent aux environs $d u 45^{e} j$, les $1^{r e s}$ gousses vers le $50^{e} j$. Le remplissage des grains débute vers le $70^{e} j$.

Each value is the mean of 4 replicates per symbiosis, at each sampling date. The $1^{\text {st }}$ flowers appear around the $45^{\text {th }}$ day; the $1^{\text {st }}$ pods around the $50^{\text {th }}$ day. Seed filling starts around the $70^{\text {th }}$ day.

TABLEAU 1

Influence d'un apport d'azote combiné sur la croissance et l'activité nitrogénase du soja inoculé avec la souche GA. Effect of combined nitrogen on the growth and nitrogenase activity of soybean inoculated with strain $G A$

\begin{tabular}{|c|c|c|c|c|c|c|}
\hline & \multicolumn{3}{|c|}{ Giesen } & \multicolumn{3}{|c|}{ Fengshou } \\
\hline & \multicolumn{2}{|c|}{ GA } & \multirow[t]{2}{*}{$\mathrm{PJ} 17$} & \multicolumn{2}{|c|}{ GA } & \multirow[t]{2}{*}{ PJ17 } \\
\hline & ON & $+N$ & & ON & $+\mathrm{N}$ & \\
\hline $\mathrm{g}$ MS aérienne $\mathrm{pl}^{-1}$ & $0,89^{\mathrm{a}}$ & $1,00^{\mathrm{a}}$ & $1,99^{\mathrm{b}}$ & $0,99^{\mathrm{a}}$ & $1,47^{a b}$ & $1,78^{b}$ \\
\hline g MF nodosités $\mathrm{pl}^{-1}$ & $0,58^{\mathrm{c}}$ & $0,58^{\mathrm{c}}$ & $0,48^{\mathrm{cd}}$ & $0,32^{\mathrm{d}}$ & $0,44^{c d}$ & $0,34^{\mathrm{d}}$ \\
\hline
\end{tabular}

Plantes âgées de $50 \mathrm{j}$. Moyenne de 4 répétitions. L'azote minéral est apporté sous forme de nitrate de potassium à partir du $30^{\mathrm{e}} \mathrm{j}$, à la concentration de $1,5 \mathrm{mM}$ dans la solution nutritive. Les valeurs ayant la même lettre en exposant ne sont pas significativement différentes au seuil de 5 p. 100 d'après le test de Newman et Keuls.

Plants 50 days old. Mean of 4 replicates. Mineral nitrogen supplied as $1.5 \mathrm{mM} \mathrm{KNO}_{3}$ since the $30^{\text {th }}$ day. Values with similar exponent letter are not significantly different $(P=0.05)$ in the Newman-Keuls test.

Ces niveaux d'activité, bien que significatifs, sont cependant inférieurs à ceux enregistrés avec la même méthode de mesure sur des plantes cultivées au champ (DREVON, 1984).

\section{Efficacité relative apparente et incorporation d'azote ${ }^{15} \mathbf{N}$}

L'ERa des nodosités formées par GA est voisine de 0,50 (tabl. 2). Elle varie selon le cultivar de la plante hôte ; pour un même cultivar, elle n'est pas significa- tivement différente de l'ERa des nodosités formées par la souche PJ17. Il a été vérifié qu'il n'y a aucune activité hydrogénase dans les bactéroïdes extraits de ces nodosités.

$\mathrm{Au} 50^{\mathrm{e}} \mathrm{j}$, il n'y a pas de différence significative entre les niveaux d'incorporation d'azote ${ }^{15} \mathrm{~N}$ par les nodosités formées par GA et par PJ17. Les quantités d'azote incorporées sont de l'ordre de $0,3 \mu$ mole d'azote moléculaire/h/plante, y compris chez le cultivar « Giesen » qui, à cette date, a ses feuilles chlorosées (tabl. 3). 
TABLEAU 2

Activité nitrogénase (ARA) et efficacité relative apparente (ERa) des nodosités formées par les souches Rhizobium GA ou PJI7 sur 3 cultivars de soja.

Nitrogenase activity (ARA) and apparent relative efficiency (aRE) of the nodules formed by Rhizobium strains GA or PJI7 on 3 soybean cultivars.

\begin{tabular}{llllllll}
\hline & \multicolumn{3}{c}{ Giesen } & & 106-17 & & \multicolumn{2}{c}{ Fengshou } \\
\cline { 2 - 8 } & GA & PJ17 & GA & PJ17 & GA & PJ17 \\
\hline ARA & $3,89^{\mathrm{a}}$ & $3,05^{\mathrm{ab}}$ & $2,23^{\mathrm{ab}}$ & $2,78^{\mathrm{ab}}$ & $2,11^{\mathrm{b}}$ & $2,44^{\mathrm{ab}}$ \\
ERa & $0,3^{\mathrm{c}}$ & $0,51^{\mathrm{c}}$ & $0,60^{\mathrm{cd}}$ & $0,7^{\mathrm{d}}$ & $0,65^{\mathrm{d}}$ & $0,60^{\mathrm{cd}}$ & \\
\hline \hline
\end{tabular}

L'ARA est exprimée en $\mu$ mole $\mathrm{C}_{2} \mathrm{H}_{2}$ réduit $\mathrm{h}^{-1} \mathrm{pl}^{-1}$. Le résultat exprimé est la moyenne de 3 mesures réalisées entre 45 et $60 \mathrm{j}$ après le semis, sur 4 plantes de chaque traitement à chaque date de mesure. Les valeurs ayant des lettres différentes en exposant sont significativement différentes au seuil de 5 p. 100 d'après le test de Newman et Keuls.

ARA expressed in $\mu$ mole $\mathrm{C}_{2} \mathrm{H}_{2}$ reduced $\mathrm{h}^{-1} \mathrm{pl}^{-1}$. Means of 3 measurements made between 45 and 60 days after sowing, on 4 plants for each treatment at each date of measurement. Values with different exponent letter are significantly different $(P=0.05)$ in the Newman-Keuls test.

TABLEAU 3

Activité nitrogénase (ARA) et incorporation d'azote ${ }^{15} \mathrm{~N}$ des nodosités formées par les souches Rhizobium $G A$ ou PJI7 sur 3 cultivars de soja.

Nitrogenase activity (ARA) and ${ }^{15} \mathrm{~N}$ incorporation of nodules formed by Rhizobium strains $G A$ or PJ17 on 3 soybean cultivars.

\begin{tabular}{|c|c|c|c|c|c|c|}
\hline & \multicolumn{2}{|c|}{ Giesen } & \multicolumn{2}{|c|}{$106-17$} & \multicolumn{2}{|c|}{ Fengshou } \\
\hline & GA & PJ17 & GA & PJ17 & GA & $\mathrm{PJ} 17$ \\
\hline ARA & $2,16^{\mathrm{ab}}$ & $1,62^{\mathrm{ab}}$ & $2,16^{\mathrm{ab}}$ & $2,82^{b}$ & $1,97^{a b}$ & $1,47^{\mathrm{a}}$ \\
\hline Incorporation de ${ }^{15} \mathrm{~N}_{2}$ & $0,37^{\mathrm{d}}$ & $0,31^{\mathrm{cd}}$ & $0,24^{\mathrm{cd}}$ & $0,16^{\mathrm{c}}$ & $0,24^{\mathrm{cd}}$ & $0,37^{\mathrm{d}}$ \\
\hline
\end{tabular}

L'ARA est exprimée en $\mu$ mole $\mathrm{C}_{2} \mathrm{H}_{2}$ réduit $\mathrm{h}^{-1} \mathrm{pl}^{-1}$ et l'incorporation de l'azote ${ }^{15} \mathrm{~N}$ en $\mu$ mole $\mathrm{N}_{2}$ réduit $\mathrm{h}^{-1} \mathrm{pl}^{-1}$. Les plantes sont âgées de $50 \mathrm{j}$. Les résultats exprimés sont la moyenne de 6 répétitions par traitement. Les valeurs ayant des lettres différentes en exposant sont significativement différentes au seuil de 5 p. 100 d'après le test de Newman et Keuls.

ARA expressed in $\mu$ mole $\mathrm{C}_{2} \mathrm{H}_{2}$ reduced $\mathrm{h}^{-1} \mathrm{pl}^{-1}$ and ${ }^{15} \mathrm{~N}$ incorporation in mole $\mathrm{N}_{2}$ reduced $\mathrm{h}^{-1} \mathrm{pl}^{-1}$. Plants 50 days old. Means of 6 replicates per treatment. Values with different exponent letter are significantly different $(P=0.05)$ in the Newman-Keuls test.

\section{DISCUSSION}

La souche GA à croissance rapide isolée au Vietnam est moins effective (effet sur la croissance de la plante hôte) en symbiose avec le soja que la souche à croissance lente PJ17. La différence d'effectivité entre les 2 souches dépend néanmoins du cultivar. Elle est la plus forte avec " Giesen » dont l'inoculation avec GA se traduit par un ralentissement de plus de 50 p. 100 de la croissance et une chlorose du feuillage. Par contre, chez «106-17 » et surtout chez « Fengshou ", cultivar d'origine asiatique, ces effets dus à l'inoculation avec GA sont moins prononcés.

Cette moindre effectivité de la souche GA ne semble pas être due à une incapacité de cet isolat à fixer l'azote en symbiose avec le soja. En effet, les masses des nodosités formées par chacune des souches GA et PJ17, sont comparables sur chaque cultivar jusqu'au $60^{\mathrm{e}} \mathrm{j}$. Les mesures d'ARA sont également comparables, ce qui indique que ces nodosités ont des niveaux voisins d'activité nitrogénase. Cependant, l'ARA n'est qu'une estimation du flux d'électrons transférés par la nitrogénase : elle ne permet pas de distinguer la part du pouvoir réducteur qui est effectivement allouée à la réduction de l'azote, de la part qui est utilisée pour la réduction des protons (DREVON \& SALSAC, 1984), parts qui peuvent varier dans une large proportion (SCHUBERT \& EVANS, 1976). C'est pourquoi les quantités d'hydrogène émises par les nodosités ont été mesurées. Les ERa calculées à partir de ces données indiquent qu'environ 50 p. 100 des électrons transférés par la nitrogénase dans les symbioses avec GA sont alloués à la synthèse d'hydrogène. Le niveau correspondant d'allocation des électrons à $\mathrm{N}_{2}$ est comparable à celui observé généralement sur les associations de légumineuses avec des souches Hup ${ }^{-}$de Rhizobium (EVANS et al., 1981). Il en résulte que la souche GA a, dans les conditions expérimentales de ce travail, une capacité à fixer l'azote qui est comparable à celle de PJ17. Cette conclusion est confirmée par les niveaux comparables d'incorporation de l'azote ${ }^{15} \mathrm{~N}$ par les nodosités de GA et de PJ17.

La chlorose ou la relativement faible croissance des plantes en présence de GA pourraient donc s'expliquer non pas par une moindre fixation d'azote, mais par un facteur toxique sécrété par cette souche. Une substance nommée rhizobitoxine a été mise en évidence chez d'autres souches de Rhizobium qui induisent une chlorose chez la plante hôte (OWENS \& WRIGHT, 1965). Cette hypothèse semble être confirmée par l'absence d'effet d'un faible apport d'azote combiné sur la croissance de "Giesen » en présence 
de GA ainsi que par la présence de proline en abondance dans les graines (THU, résultats non publiés), ce qui est généralement l'indication d'une réaction de la plante à une situation défavorable.

La variété « Fengshou ", d'origine asiatique, serait plus résistante à cette substance, puisque son rendement en présence d'azote combiné est moins affecté que celui de "Giesen ». Dans une moindre mesure, la variété «106-17" apparaît être résistante. Ceci infirme les observations précédentes (KEYSER et al., 1982) d'après lesquelles les variétés d'origine américaine, ce qui est le cas de 106-17 qui est une sélection française à partir de cultivars américains (VIDAL, comm. pers.), sont chlorosées en présence des souches à croissance rapide de Rhizobium isolées sur le continent asiatique.

Il apparaît par conséquent possible d'obtenir des symbioses effectives entre des variétés de soja adaptées aux conditions européennes de culture et des souches de Rhizobium à croissance rapide. Cet objectif exige de tester un plus grand nombre de variétés de soja inoculées avec GA ainsi que d'obtenir d'autres isolats de Rhizobium à croissance rapide capables de noduler le soja. Parallèlement, la compréhension du déterminisme de la plus faible effectivité de GA observée ici devrait fournir des moyens supplémentaires pour la sélection d'associations soja-Rhizobium à croissance rapide, ayant une capacité élevée à fixer l'azote atmosphérique.

Reçu le 9 novembre 1984. Accepté le 3 octobre 1985.

\section{REMERCIEMENTS}

Les auteurs remercient MM. NGo ThE DAN (I.N.S.A., Hanoï) et H. J. Evans (O.S.U., Corvallis) pour leur avoir fourni respectivement les souches GA et PJ17 ; ainsi que A. Gojon (I.N.R.A. Montpellier) pour sa contribution au dosage d'azote ${ }^{15} \mathrm{~N}$ dans les nodosités; ainsi que A. VIDAL (I.N.R.A. Montpellier) pour leur avoir fourni les graines de trois cultivars de soja.

\section{RÉFERENCES BIBLIOGRAPHIQUES}

Drevon J. J., Tillard P., Salsac L., 1983. Influence de deux espèces de légumineuses, Vigna radiata et Vigna unguiculata sur l'activité hydrogénase de la souche CB 756 de Rhizobium du groupe " cowpea ». C. R. Acad. Sci., Paris, 296, 979-982.

Drevon J. J., 1984. Relations entre le métabolisme de l'hydrogène et la fixation de l'azote dans la symbiose Phaseoleae-Rhizobium. Thèse Doct.-Ing., USTL, Montpellier, $200 \mathrm{p}$.

Drevon J. J., Salsac L., 1984. Relations entre le métabolisme de l'hydrogène et la fixation d'azote par les nodosités de légumineuse. Phvsiol. V'ég., 22, 263-275.

Evans H. J., Purohit K., Cantrell M. A., Eisbrenner G., Russell S. A., Hanus F. J., Lepo J. E., 1981. Hydrogen losses and hydrogenases in nitrogen fixing organisms. In Gibson A. H. \& Newton W. E. : "Current Perspectives in Nitrogen Fixation". Griffin Press Ltd., pp. 84-96.

Guiraud G., Fardeau J. C., 1980. Détermination isotopique par spectrométrie optique de composés faiblement enrichis en azote 15. Anal'sis, 8, 148-152.

Keyser H. K., Bohlool B. B., Weber D. F., 1982. Fast growing Rhizobia isolated from root nodules of soybean. Science, 215, 16311632 .
Kimou A., 1984. Influence de l'azote combiné et du recyclage de l'hydrogene sur l'activité et l'efficacité relative de la symbiose Glycine max.-Rhizobium japonicum. Thèse Doct.-Ing., ENSAM, Montpellier, $126 \mathrm{p}$.

Lepo J. E., Hickock R. E., Cantrell M. A., Russell S. A., Evans H. J., 1981. Revertible hydrogen uptake deficient mutants of Rhizobium japonicum. J. Bacteriol., 146, 614-620.

Owens L. D., Wright D. A., 1965. Production of the soybeanchlorosis toxin by Rhizobium japonicum in pure culture. Plant physiol. Veg., 17, 55-66.

Robin P., 1979. Etude de quelques conditions d'extraction de la' nitrate réductase des racines et des feuilles de plantules de maïs. Physiol. Veg., 17, 55-66.

Schubert K. R., Evans H. J., 1976. Hydrogen evolution a major factor affecting the efficiency of nitrogen fixation in nodulated symbionts. Proc. Nat. Sci. USA, 73, 1207-1211.

Vincent J. M., 1970. A manual for the practical study of root nodule bacteria. Blackwell Science Publisher. $120 \mathrm{p}$. 